\title{
Effects of Carbon Nanotube Structure, Purity, and Alignment on the Heat Conduction Properties of Carbon Films and Fibers
}

\author{
Junjie Chen *
}

How to cite this paper: Chen, J. (2022). Effects of Carbon Nanotube Structure, Purity, and Alignment on the Heat Conduction Properties of Carbon Films and Fibers. Online Journal of Materials Science, 1(1), 1-11. Retrieved from

https://www.scipublica-

tions.com/journal/index.php/materials/article/view/248

Received: January 3, 2022

Accepted: February 23, 2022

Published: February 24, 2022

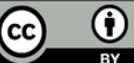

Copyright: (๑) 2022 by the authors. Submitted for possible open access publication under the terms and conditions of the Creative Commons Attribution (CC BY) license (http://creativecommons.org/licenses /by/4.0/).
Department of Energy and Power Engineering, School of Mechanical and Power Engineering, Henan Polytechnic University, 2000 Century Avenue, Jiaozuo, Henan, 454000, P.R. China

*Correspondence: cjjmmm@163.com

\begin{abstract}
The increasing popularity of carbon nanotubes has created a demand for greater scientific understanding of the characteristics of thermal transport in nanostructured materials. However, the effects of impurities, misalignments, and structure factors on the thermal conductivity of carbon nanotube films and fibers are still poorly understood. Carbon nanotube films and fibers were produced, and the parallel thermal conductance technique was employed to determine the thermal conductivity. The effects of carbon nanotube structure, purity, and alignment on the thermal conductivity of carbon films and fibers were investigated to understand the characteristics of thermal transport in the nanostructured material. The importance of bulk density and cross-sectional area was determined experimentally. The results indicated that the prepared carbon nanotube films and fibers are very efficient at conducting heat. The structure, purity, and alignment of carbon nanotubes play a fundamentally important role in determining the heat conduction properties of carbon films and fibers. Single-walled carbon nanotube films and fibers generally have high thermal conductivity. The presence of non-carbonaceous impurities degrades the thermal performance due to the low degree of bundle contact. The thermal conductivity may present power law dependence with temperature. The specific thermal conductivity decreases with increasing bulk density. At room temperature, a maximum specific thermal conductivity is obtained but Umklapp scattering occurs. The specific thermal conductivity of carbon nanotube fibers is significantly higher than that of carbon nanotube films due to the increased degree of bundle alignment.
\end{abstract}

Keywords: Carbon nanotubes; Thermal properties; Carbon fibers; Thermal conductivity; Nanostructured materials; Umklapp scattering

\section{Introduction}

Carbon nanotubes can exhibit unique ability to conduct heat [1, 2], referred to as heat conduction properties. Specifically, carbon nanotubes are a highly effective thermal conductor in the longitudinal direction. However, a thermal barrier is formed in the radial direction. For an individual single-walled carbon nanotube, the thermal conductivity in the radial direction is around $1.52 \mathrm{~W} /(\mathrm{m} \cdot \mathrm{K})$ at room temperature [3]. In contrast, carbon nanotubes show superior heat conduction properties along the longitude directions. The thermal conductivity in the longitudinal direction is around $3500 \mathrm{~W} /(\mathrm{m} \cdot \mathrm{K})$ at room temperature [4]. Consequently, carbon nanotubes outperform diamond as the best thermal conductor. When macroscopic, ordered assemblies of single-walled carbon nanotubes are formed, the thermal conductivity of carbon nanotube films and fibers could reach up to around $1500 \mathrm{~W} /(\mathrm{m} \cdot \mathrm{K})$ at room temperature [5]. The heat conduction properties of carbon nanotube networks vary significantly, with a minimum of thermal conductivity less than $0.1 \mathrm{~W} /(\mathrm{m} \cdot \mathrm{K})[6]$. The heat conduction properties depend on a variety of factors such as impurities and misalignments. Single-walled carbon nanotube are stable up to around $1000 \mathrm{~K}$ in air and around $3000 \mathrm{~K}$ in vacuum [7]. The study of heat transport phenomena 
involved in carbon nanotubes is an active area of interest [8] due to the potential for applications in thermal management.

The thermal conductivity of carbon nanotubes depends heavily upon crystallographic defects. Phonons can scatter due to crystallographic defects. This will lead to the increased relaxation rate, thereby decreasing thermal conductivity associated with the reduced mean free path of phonons $[9,10]$. In single-walled carbon nanotubes, the mean free path varies from $50 \mathrm{~nm}$ to $1500 \mathrm{~nm}$ [11, 12]. Crystallographic defects will lead to a significant reduction in mean free path [13], for example, $4 \mathrm{~nm}$ or less [14]. The thermal conductivity of carbon nanotubes depends also upon the structure of the nanotubes. The thermal conductance of multi-walled carbon nanotubes is significantly higher than the sum of that of each individual shell due to the inter-wall interactions [15]. The thermal conductivity of a multi-walled carbon nanotube is lower than that of a single-walled carbon nanotube with an identical diameter configuration [16] due to an increase in crosssectional area.

Bulk carbon nanotubes can be used as composite fibers in polymers, but the bulk structure will reduce the ability to conduct heat, causing a decrease in thermal conductivity. The thermal conductivity of carbon nanotube fibers is comparable to that of common metals [17]. Through chemical modification, however, the ability to conduct heat is comparable to or higher than that of highly conductive metals [18], for example, copper. The thermal conductivity varies significantly depending upon the density and cross-sectional area of the bulk material. The bulk carbon nanotube material contains pores $[19,20]$. Consequently, the thermal conductivity of the non-compact bulk material is much lower than that of the skeletal material, since the bulk volume is inclusive of the void fraction. The skeletal portion of the bulk material is often referred to as the "matrix" or "frame". However, there is considerable uncertainty in determination of the density of the bulk material. Consequently, the effects of carbon nanotube structure, purity, and alignment on the thermal conductivity of carbon films and fibers are still poorly understood. Little research has been conducted to determine which parameters are important for high thermal conductivity.

This study relates to the heat conduction properties of carbon nanotube films and fibers. Carbon nanotube films and fibers were produced, and the parallel thermal conductance technique was employed to determine the thermal conductivity. The effects of carbon nanotube structure, purity, and alignment on the thermal conductivity of carbon films and fibers were investigated to understand the characteristics of thermal transport in the nanostructured material. The objective is to gain insight into the fundamental characteristics of thermal transport in carbon nanotubes. Particular emphasis is placed on the dependence of thermal conductivity on carbon nanotube structure, purity, and alignment, with an attempt to improve the heat conduction properties for carbon nanotube films and fibers.

\section{Experimental methods}

\subsection{Preparation of carbon nanotube films and fibers}



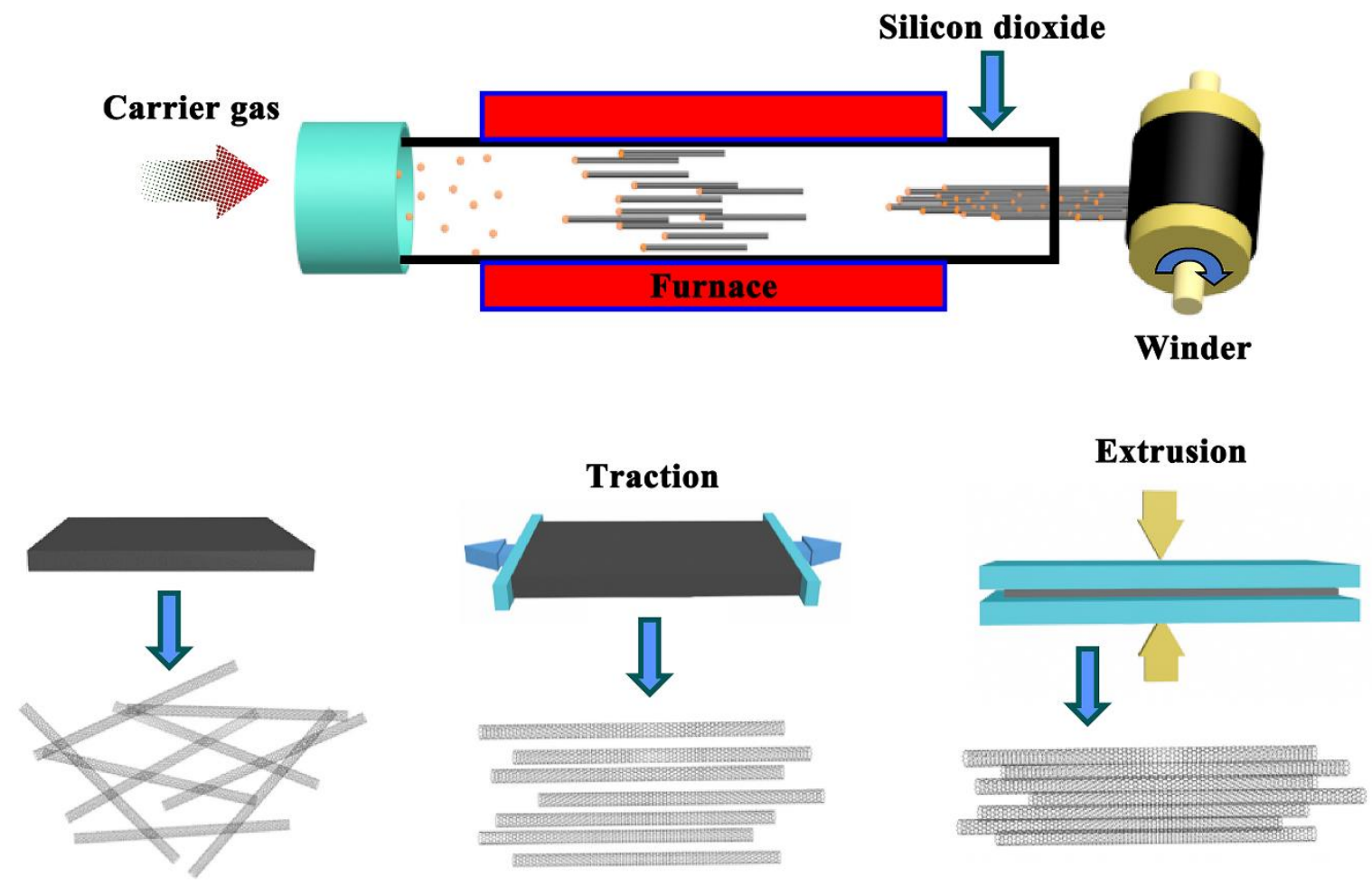

Figure 1. Schematic illustration of the preparation process of carbon nanotube films. A dense film was laid down with aligned carbon nanotube bundles.

Carbon nanotube films were produced by spinning continuously onto a single rotational winder of a spinning machine. The preparation process of carbon nanotube films is depicted schematically in Figure 1. A dense film was laid down with aligned carbon nanotube bundles. The dense film was sprayed with acetone to further condense the carbon nanotube networks. By preparing a dense film in such a manner, the carbon nanotubes were highly aligned [21], although the surface tension effect is significant in the extrusion process [22]. A laser was used to cut the carbon nanotube film into small pieces for the measurement of thermal conductivity. Carbon nanotube fibers were produced by stretching the dense film with two rotational winders of the spinning machine. The two rotational winders operate with only a small difference in rotation rate.

\subsection{Thermal conductivity measurement methods}

To determine the thermal conductivity, the parallel thermal conductance technique was employed [23, 24], since the diameter of the needle-like samples was very small. Fourier's Law was used to compute the thermal conductivity. This steady-state method has been carried out to measure the thermal conductivity of boron nitride nanotube sheets [25], and carbon nanotube sheets [26] and yarns [27, 28]. The configuration of the parallel thermal conductance technique is depicted schematically in Figure 2. A preliminary measurement of thermal conductance was performed with respect to the sample holder itself so as to determine the background or base-line heat conduction and losses associated with the sample stage. The sample was attached and the thermal conductance of the system was measured. The parallel thermal conductance can be obtained by subtraction. In this method, all conductance factors arising from the thermal contacts, sample, and thermal radiation from the sample accounted for. The radiative heat losses were caused primarily by the thermal radiation from the hot surface of the heater. However, such heat losses were already included in the base line. Therefore, a correction factor of 0.5 was introduced into the method $[23,24]$ to account for the radiative heat losses. 


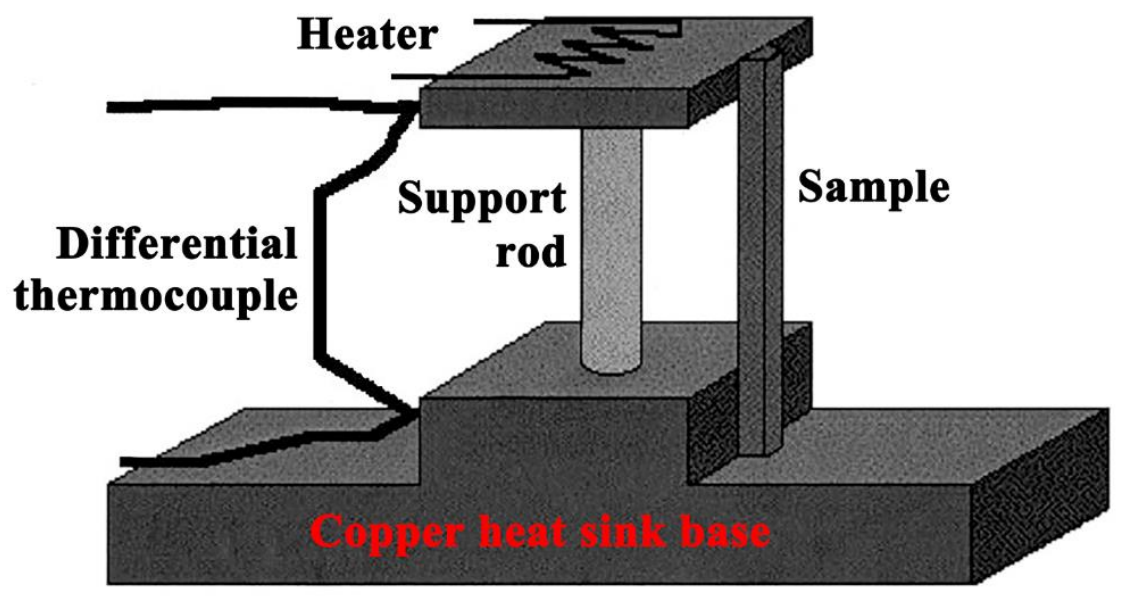

Figure 2. Schematic illustration of the configuration of the parallel thermal conductance technique. The base line was measured with the sample holder itself and then the sample was attached to perform the measurement of parallel thermal conductance in which the base line was subtracted.

\subsection{Linear mass density measurement methods}

The linear mass density of the carbon nanotube films was measured by using a gravimetric method [29]. In contrast, the linear mass density of the carbon nanotube fibers was measured by using a vibroscope method $[29,30]$. The sample was tensioned between two hard points of a mechanical vibroscope. Mechanical vibration was induced and the fundamental frequency was measured. The specific thermal conductivity is defined as the thermal conductivity normalized by bulk density. The bulk density can be determined in terms of the linear mass density and the cross-sectional area.

\section{Results and discussion}

\subsection{Material characterization}

The prepared carbon nanotube fibers are characterized by scanning electron microscopy. The surface morphology is analyzed with scanning electron microscope SU3800, Hitachi High-Tech Corporation. Scanning electron microscopy images are presented in Figure 3 for the carbon nanotube fibers prepared by the method described above. The carbon nanotubes are highly aligned. However, the fiber material contains a relatively small amount of short deformed carbon nanotubes. The carbon nanotubes are of the order of several hundred microns.
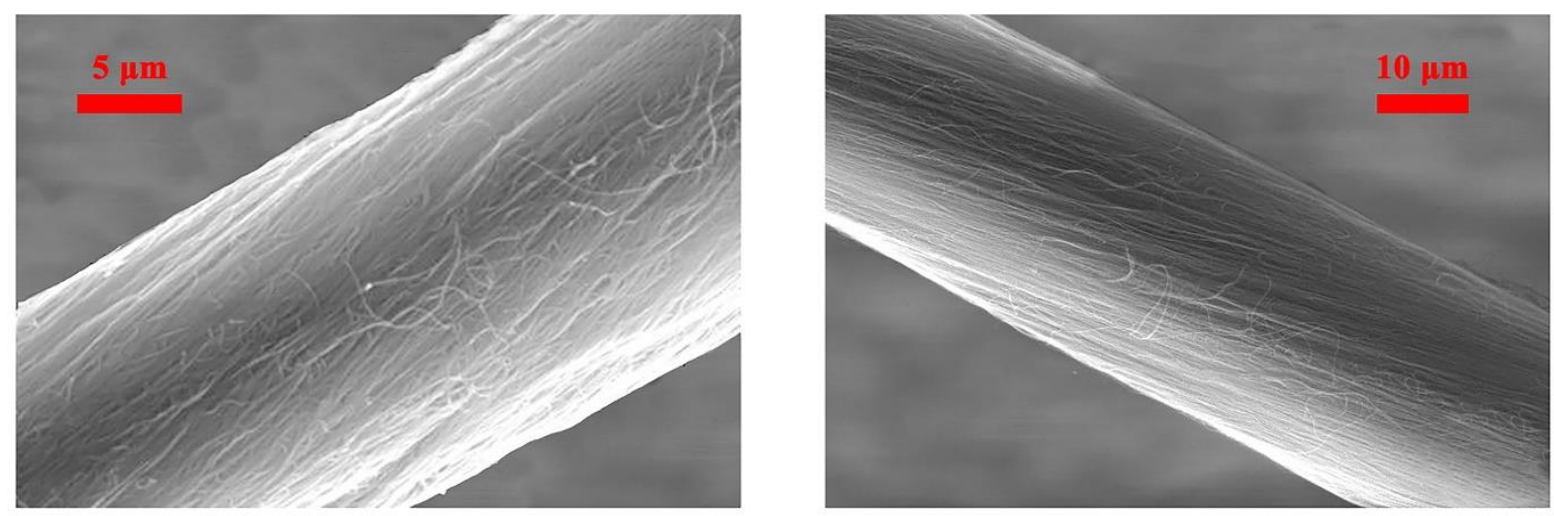

Figure 3. Scanning electron microscopy images of the carbon nanotube fibers prepared by the method described in detail above. 
Four samples are prepared with different structure, purity, and alignment in order to investigate the effects of various factors on the thermal conductivity. Three structural parameters are selected in terms of alignment, purity, and the number of walls. The structural parameter space for the selection of samples is illustrated in Figure 4 in the style of the design of measurement experiments. For type A, the carbon nanotube films or fibers are composed mostly of multi-walled carbon nanotubes. In addition, there is a small amount of short deformed carbon nanotubes. For type B, a mixture of carbon nanotubes is used. More specifically, the carbon nanotube films or fibers are composed of singlewalled and multi-walled carbon nanotubes. In addition, there is a small amount of amorphous carbon. For type $\mathrm{C}$, the carbon nanotube films or fibers are composed mostly of single-walled carbon nanotubes. In addition, there is a small amount of non-carbonaceous impurities. For type D, the carbon nanotube films or fibers are composed of single-walled carbon nanotubes with very high purity. The understanding of which parameters are important for high thermal conductivity is made possible through the comparison of heat conduction properties between the nanostructured materials.

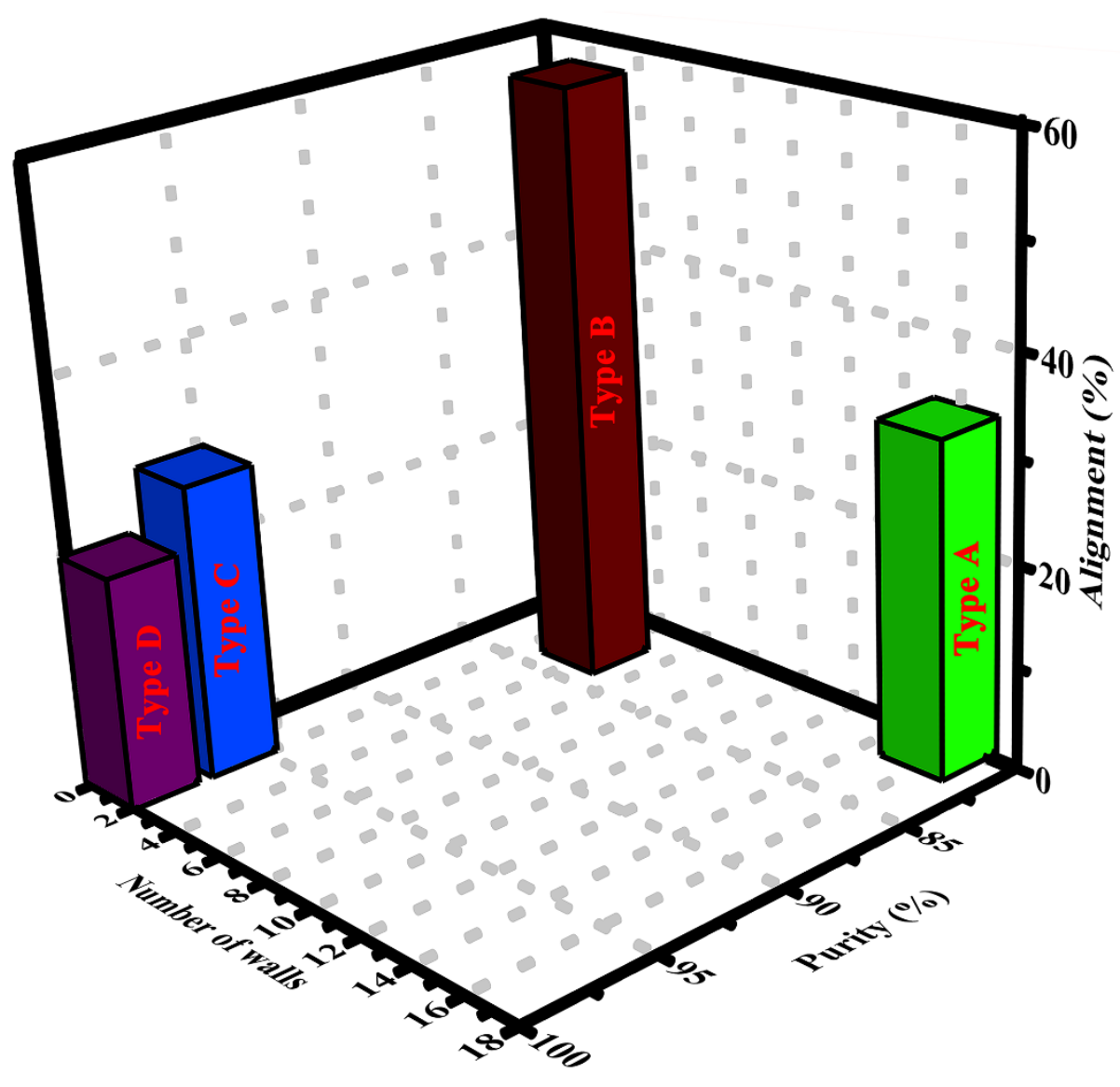

Figure 4. Structural parameter space for the selection of samples in the style of the design of measurement experiments.

\subsection{Thermal conductivity of carbon nanotube films}

The effects of structure, purity, and alignment on the specific thermal conductivity of carbon nanotube films are illustrated in Figure 5 at different temperatures. The specific thermal conductivity of the A-type film is lower than that of the other-type films. Multiwalled carbon nanotubes generally have low thermal conductivity. The A-type film is composed mostly of multi-walled carbon nanotubes. Therefore, the specific thermal conductivity is lower than that of the film composed of single-walled carbon nanotubes. The 
specific thermal conductivity of the C-type film is higher than that of the A-type film, since the structure is different from each other. The structural variation will lead to the difference in thermal conductivity. However, the specific thermal conductivity of the C-type film is much lower than that of the D-type film. This is because the presence of non-carbonaceous impurities reduces the degree of bundle contact between single-walled carbon nanotubes, which increases the thermal resistance at the interface junctions and degrades the thermal performance of the C-type film.

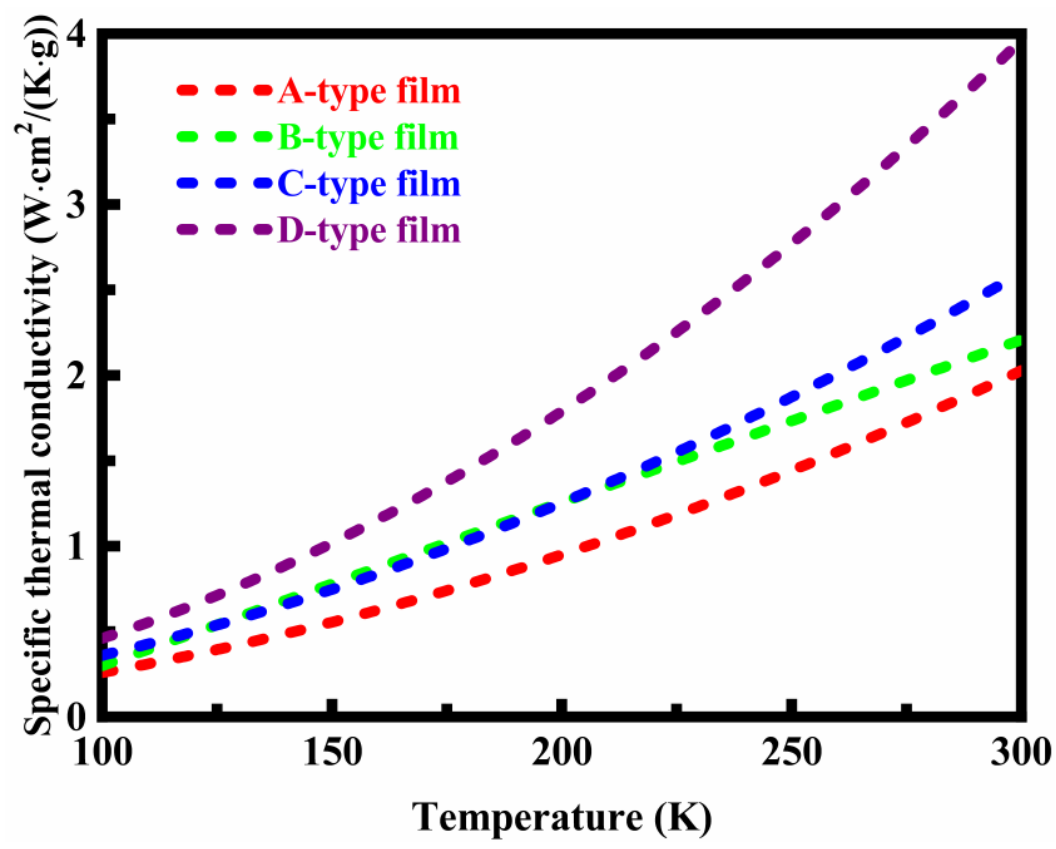

Figure 5. Specific thermal conductivity of the carbon nanotube films at different temperatures. There exist significant differences in terms of structure, purity, and alignment between the films.

While the specific thermal conductivity increases with temperature, the effect of temperature is different for the B-type film and the other-type films. The specific thermal conductivity of the B-type film has a linear relationship with temperature. For the other-type films, there is an exponential increase in specific thermal conductivity. The effect of temperature on thermal conductivity is illustrated in Figure 6 for the carbon nanotube films. The thermal conductivity of all the films presents power law dependence with temperature except that of the B-type film. The power law index is about 1.87, 1.80, and 1.96 for the thermal conductivity of the A, C, and D type films, respectively. In contrast, the temperature index is 0.99 for the thermal conductivity of the B-type film. The temperature index is small, which reduces the dimensionality of the propagation of phonons [31,32] due to an increase in the degree of bundle alignment and contact. 


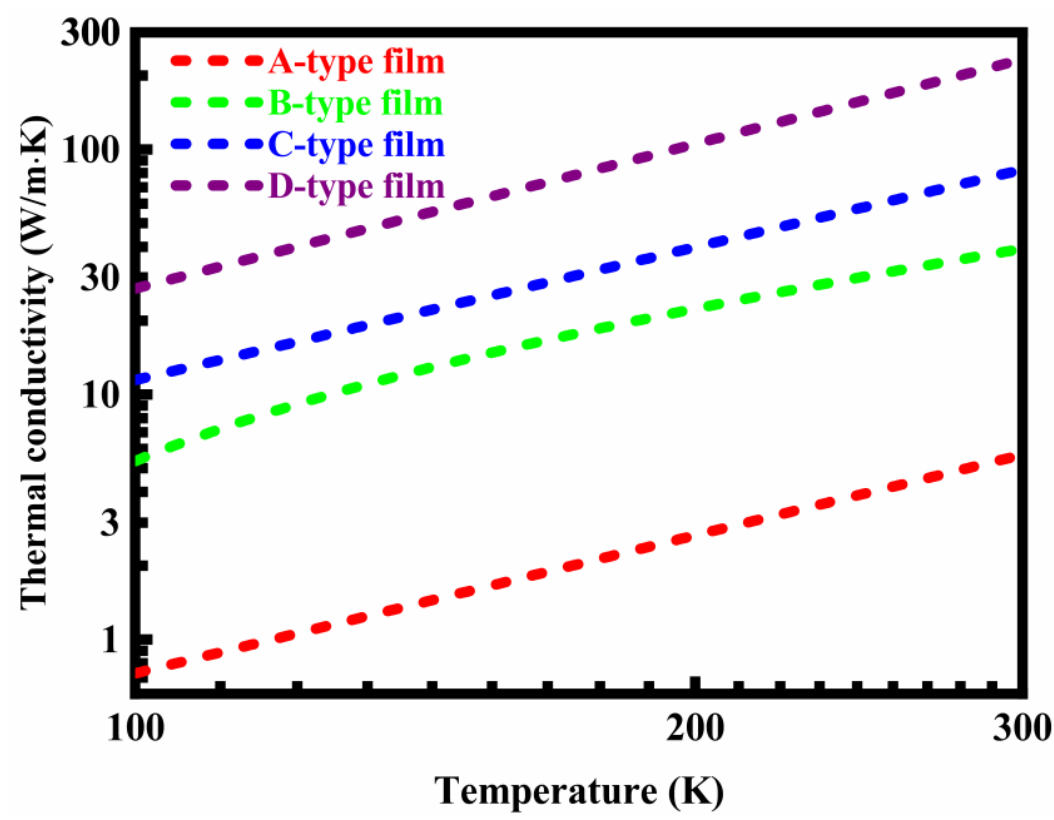

Figure 6. Thermal conductivity of the carbon nanotube films at different temperatures. The thermal conductivity is indicated with points plotted on a logarithmic coordinate system.

The effect of bulk density on the specific thermal conductivity at room temperature is illustrated in Figure 7 for the carbon nanotube films. At room temperature, the highest specific thermal conductivity is achieved for the D-type film. In addition, the power law index is largest, although the degree of bundle alignment is very low, which reduces the mechanical stiffness of the film. At room temperature, the lowest specific thermal conductivity is obtained for the A-type film. A tentative explanation could be made for the distinctive phenomenon. The specific thermal conductivity decreases with increasing bulk density. The low bulk density of the D-type film compensates for the adverse effect of poor alignment on specific thermal conductivity. Therefore, the bulk density may be controlled to produce carbon nanotube films with high thermal conductivity.

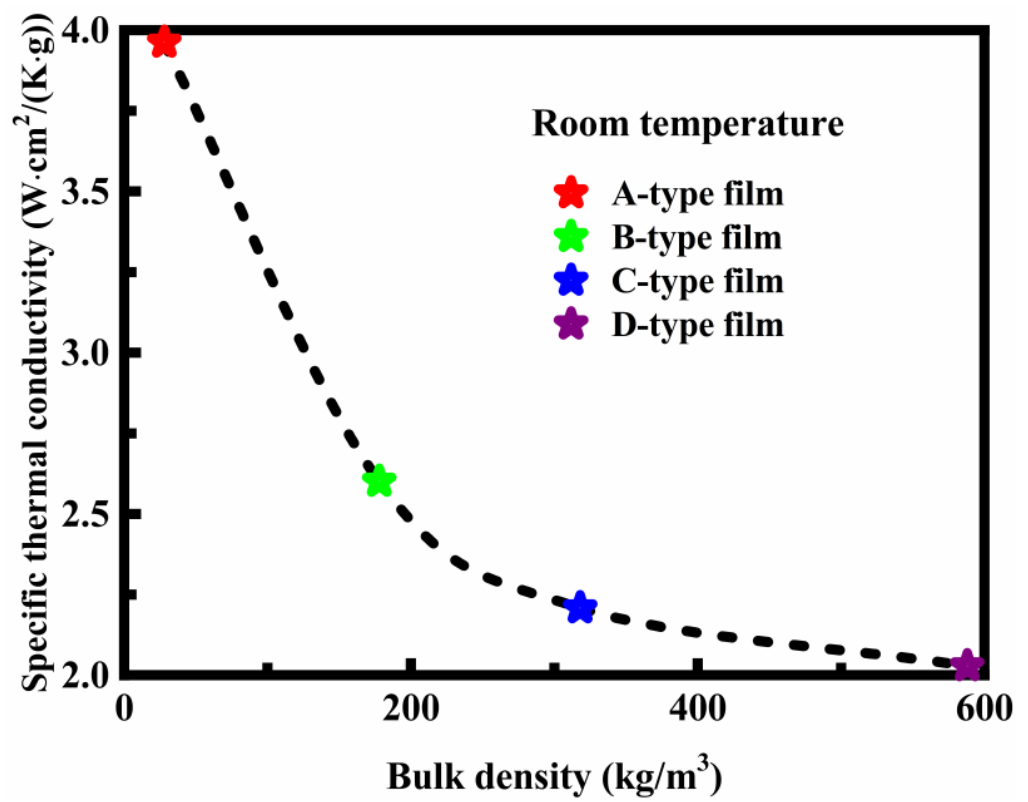

Figure 7. Effect of bulk density on the specific thermal conductivity of the carbon nanotube films at room temperature. 


\subsection{Thermal conductivity of carbon nanotube fibers}

The effects of structure, purity, and alignment on the specific thermal conductivity of carbon nanotube fibers are illustrated in Figure 8 at different temperatures. The specific thermal conductivity of the fibers is around $10 \mathrm{~W} \cdot \mathrm{cm}^{2} /(\mathrm{K} \cdot \mathrm{g})$ at room temperature, which is comparable to or higher than that of pristine and chemically modified carbon nanotube fibers $[17,18]$. A maximum specific thermal conductivity is obtained at room temperature, which appears as a peak in the dependence of temperature in Figure 8, as determined previously $[18,32]$. The maximum specific thermal conductivity represented by the peaks indicates the onset of Umklapp scattering. The Umklapp scattering is the dominant process for thermal resistivity, which limits the specific thermal conductivity. High thermal conductivity of carbon fibers is often associated with a high modulus of elasticity [33, 34]. Therefore, the carbon nanotube fibers have sufficient strength to be used as a reinforcement for composite materials. Clearly, the specific thermal conductivity of the fibers is significantly higher than that of the films. This is caused by the improved degree of bundle alignment for the fibers. A high degree of bundle alignment will lead to an increase in thermal conductivity due to the increased interfacial area between the carbon nanotubes.

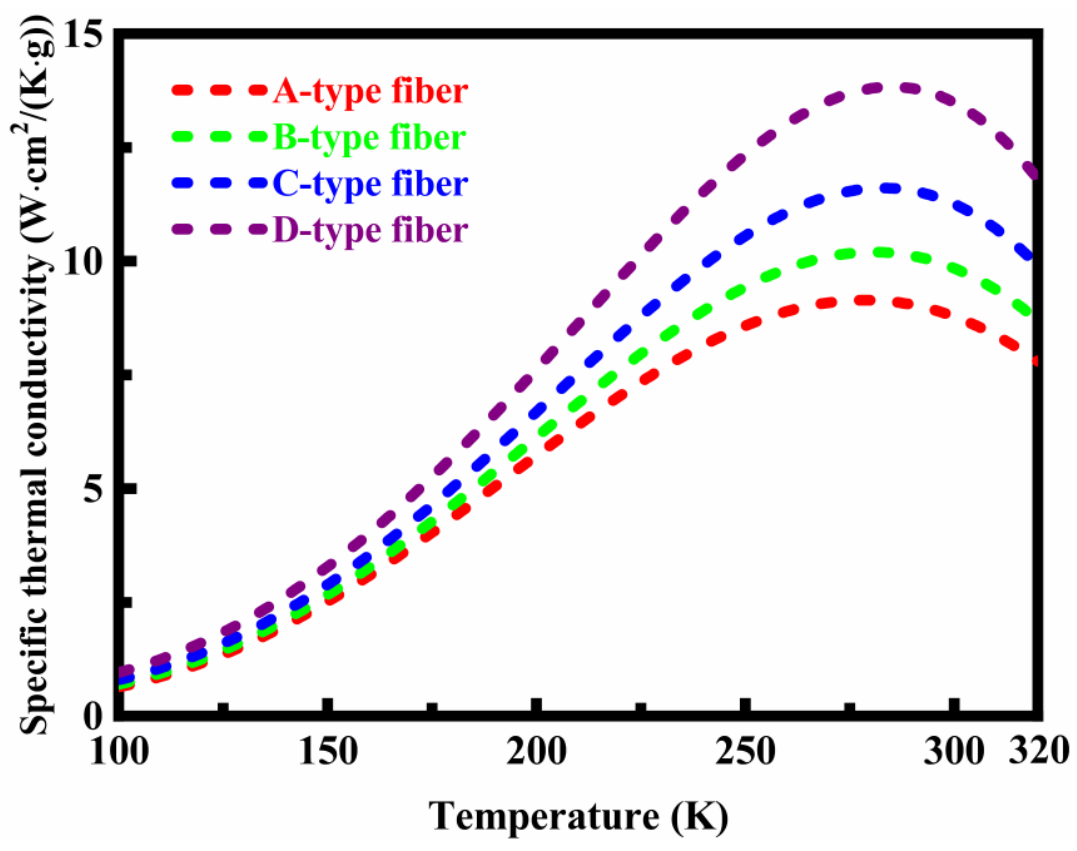

Figure 8. Specific thermal conductivity of the carbon nanotube fibers at different temperatures. There exist significant differences in terms of structure, purity, and alignment between the fibers.

The effect of cross-sectional area on the specific thermal conductivity at room temperature is illustrated in Figure 9 for the carbon nanotube films and fibers. Some previous data are also included for comparison. These previous data are available in the literature $[18,27,28,35,36,37,38]$. The fibers have higher specific thermal conductivity than the films. Under the same cross-sectional area conditions, the specific thermal conductivity of the films and fibers is higher than that previously reported in the literature. The carbon nanotubes are of the order of several hundred microns, which is significantly greater than the phonon mean free path. As the length of carbon nanotubes increases, the effect of temperature on thermal conductivity becomes more pronounced $[4,38]$. The length of the carbon nanotubes contained in the films and fibers is much greater than that of the carbon nanotubes used in the literature. Consequently, the films and fibers are very efficient at conducting heat and typically have higher specific thermal conductivity in comparison to the previous data. 


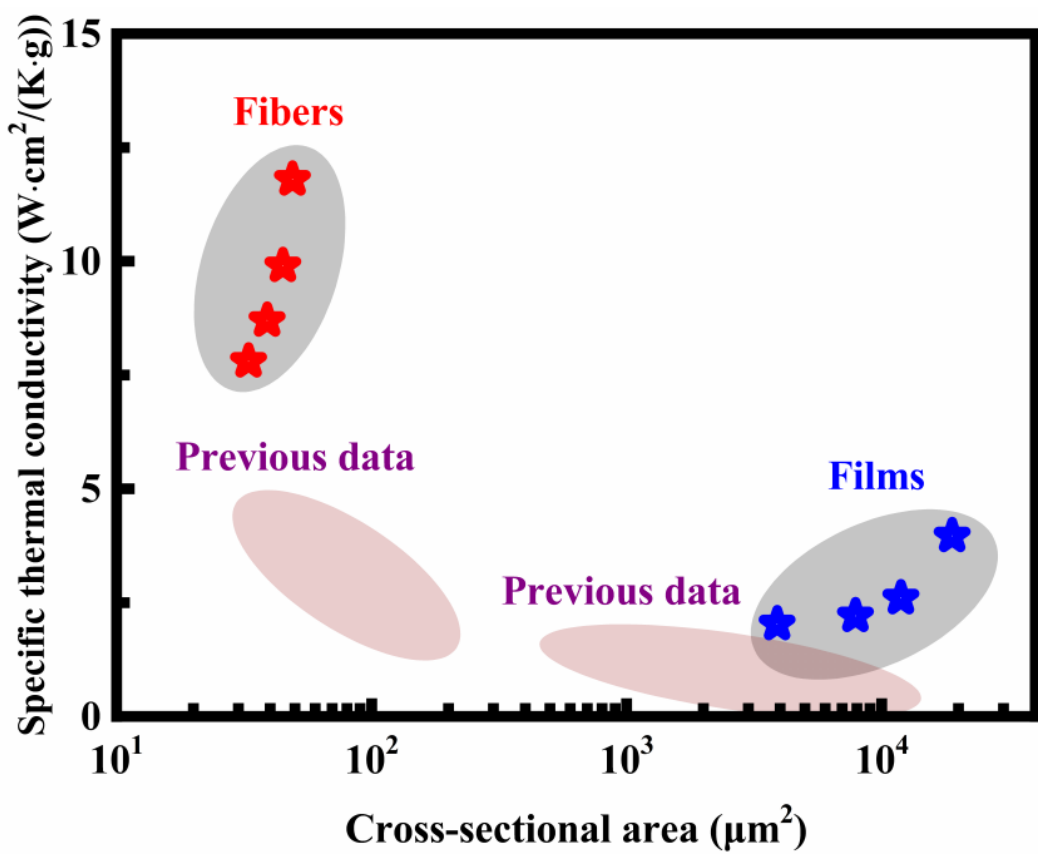

Figure 9. Effect of cross-sectional area on the specific thermal conductivity of the carbon nanotube films and fibers at room temperature. Some previous data are also included for comparison.

\section{Conclusions}

Carbon nanotube films and fibers were produced, and the parallel thermal conductance technique was employed to determine the thermal conductivity. The effects of carbon nanotube structure, purity, and alignment on the heat conduction properties of carbon films and fibers were investigated to understand the characteristics of thermal transport in the nanostructured material. The major conclusions are summarized as follows:

- Multi-walled carbon nanotube films and fibers generally have low thermal conductivity.

- The presence of non-carbonaceous impurities reduces the degree of bundle contact between carbon nanotubes, thereby increasing the thermal resistance at the interface junctions and degrading the thermal performance.

- The thermal conductivity presents power law dependence with temperature or has a linear relationship with temperature.

- The specific thermal conductivity decreases with increasing bulk density. Low bulk density can compensate for the adverse effect of poor alignment on specific thermal conductivity.

- A maximum specific thermal conductivity is obtained at room temperature due to the onset of Umklapp scattering.

- The specific thermal conductivity of carbon nanotube fibers is significantly higher than that of carbon nanotube films. The improved thermal properties are caused by the increased degree of bundle alignment.

- The prepared carbon nanotube films and fibers are very efficient at conducting heat due to the increased length of the carbon nanotubes. 


\section{References}

[1] T.W. Ebbesen. Carbon nanotubes. Physics Today, Volume 49, Issue 6, 1996, Pages 26-32.

[2] Y. Ando, X. Zhao, T. Sugai, and M. Kumar. Growing carbon nanotubes. Materials Today, Volume 7, Issue 10, 2004, Pages 2229.

[3] K.S. Ibrahim. Carbon nanotubes-properties and applications: A review. Carbon Letters, Volume 14, Issue 3, 2013, Pages 131144.

[4] E. Pop, D. Mann, Q. Wang, K. Goodson, and H. Dai. Thermal conductance of an individual single-wall carbon nanotube above room temperature. Nano Letters, Volume 6, Issue 1, 2006, Pages 96-100.

[5] K.K. Koziol, D. Janas, E. Brown, and L. Hao. Thermal properties of continuously spun carbon nanotube fibres. Physica E: Low-dimensional Systems and Nanostructures, Volume 88, 2017, Pages 104-108.

[6] B. Kumanek and D. Janas. Thermal conductivity of carbon nanotube networks: A review. Journal of Materials Science, Volume 54, Issue 10, 2019, Pages 7397-7427.

[7] E.T. Thostenson, C. Li, and T.-W. Chou. Nanocomposites in context. Composites Science and Technology, Volume 65, Issues 34, 2005, Pages 491-516.

[8] S. Rathinavel, K. Priyadharshini, and D. Panda. A review on carbon nanotube: An overview of synthesis, properties, functionalization, characterization, and the application. Materials Science and Engineering: B, Volume 268, 2021, Article Number: 115095.

[9] N. Mingo, D.A. Stewart, D.A. Broido, and D. Srivastava. Phonon transmission through defects in carbon nanotubes from first principles. Physical Review B, Volume 77, Issue 3, 2008, Article Number: 033418.

[10] C. Sevik, H. Sevinçli, G. Cuniberti, and T. Çağın. Phonon engineering in carbon nanotubes by controlling defect concentration. Nano Letters, Volume 11, Issue 11, 2011, Pages 4971-4977.

[11] P. Kim, L. Shi, A. Majumdar, and P.L. McEuen. Thermal transport measurements of individual multiwalled nanotubes. Physical Review Letters, Volume 87, Issue 21, 2001, Article Number: 215502.

[12] C. Chang, D. Okawa, H. Garcia, A. Majumdar, and A. Zettl. Breakdown of Fourier's Law in nanotube thermal conductors. Physical Review Letters, Volume 101, Issue 7, 2008, Article Number: 075903.

[13] K. Sääskilahti, J. Oksanen, S. Volz, and J. Tulkki. Frequency-dependent phonon mean free path in carbon nanotubes from nonequilibrium molecular dynamics. Physical Review B, Volume 91, Issue 11, 2015, Article Number: 115426.

[14] T.S. Gspann, S.M. Juckes, J.F. Niven, M.B. Johnson, J.A. Elliott, M.A. White, and A.H. Windle. High thermal conductivities of carbon nanotube films and micro-fibres and their dependence on morphology. Carbon, Volume 114, 2017, Pages 160-168.

[15] X. Zhang, W.-X. Zhou, X.-K. Chen, Y.-Y. Liu, and K.-Q. Chen. Significant decrease in thermal conductivity of multi-walled carbon nanotube induced by inter-wall van der Waals interactions. Physics Letters A, Volume 380, Issue 21, 2016, Pages 18611864.

[16] B. Goh, K.J. Kim, C.-L. Park, E.S. Kim, S.H. Kim, and J. Choi. In-plane thermal conductivity of multi-walled carbon nanotube yarns under mechanical loading. Carbon, Volume 184, 2021, Pages 452-462.

[17] E. Mayhew and V. Prakash. Thermal conductivity of high performance carbon nanotube yarn-like fibers. Journal of Applied Physics, Volume 115, Issue 17, 2014, Article Number: 174306.

[18] N. Behabtu, C.C. Young, D.E. Tsentalovich, O. Kleinerman, X. Wang, A.W.K. Ma, E.A. Bengio, R.F.T. Waarbeek, J.J.D. Jong, R.E. Hoogerwerf, S.B. Fairchild, J.B. Ferguson, B. Maruyama, J. Kono, Y. Talmon, Y. Cohen, M.J. Otto, and M. Pasquali. Strong, light, multifunctional fibers of carbon nanotubes with ultrahigh conductivity. Science, Volume 339, Issue 6116, 2013, Pages 182-186.

[19] H.E. Misak and S. Mall. Investigation into microstructure of carbon nanotube multi-yarn. Carbon, Volume 72, 2014, Pages 321-327.

[20] A.S. Wu and T.-W. Chou. Carbon nanotube fibers for advanced composites. Materials Today, Volume 15, Issues 7-8, 2012, Pages 302-310.

[21] H. Zhan, Y.W. Chen, Q.Q. Shi, Y. Zhang, R.W. Mo, and J.N. Wang. Highly aligned and densified carbon nanotube films with superior thermal conductivity and mechanical strength. Carbon, Volume 186, 2022, Pages 205-214.

[22] S. Li, X. Zhang, J. Zhao, F. Meng, G. Xu, Z. Yong, J. Jia, Z. Zhang, and Q. Li. Enhancement of carbon nanotube fibres using different solvents and polymers. Composites Science and Technology, Volume 72, Issue 12, 2012, Pages 1402-1407.

[23] B.M. Zawilski, R.T. Littleton IV, and T.M. Tritt. Description of the parallel thermal conductance technique for the measurement of the thermal conductivity of small diameter samples. Review of Scientific Instruments, Volume 72, Issue 3, 2001, Pages 1770-1774.

[24] B.M. Zawilski and T.M. Tritt. Dynamic measurement access, a new technique for fast thermal conductivity measurement. Review of Scientific Instruments, Volume 72, Issue 10, 2001, Pages 3937-3939.

[25] M. Jakubinek, J.F. Niven, M.B. Johnson, B. Ashrafi, K.S. Kim, B. Simard, and M.A. White. Thermal conductivity of bulk boron nitride nanotube sheets and their epoxy-impregnated composites. physica status solidi (a) - applications and materials science, Volume 213, Issue 8, 2016, Pages 2237-2242.

[26] J.-H. Pöhls, M.B. Johnson, M.A. White, R. Malik, B. Ruff, C. Jayasinghe, M.J. Schulz, and V. Shanov. Physical properties of carbon nanotube sheets drawn from nanotube arrays. Carbon, Volume 50, Issue 11, 2012, Pages 4175-4183. 
[27] J.F. Niven, M.B. Johnson, S.M. Juckes, M.A. White, N.T. Alvarez. and V. Shanov. Influence of annealing on thermal and electrical properties of carbon nanotube yarns. Carbon, Volume 99, 2016, Pages 485-490.

[28] M.B. Jakubinek, M.B. Johnson, M.A. White, C. Jayasinghe, G. Li, W. Cho, M.J. Schulz, and V. Shanov. Thermal and electrical conductivity of array-spun multi-walled carbon nanotube yarns. Carbon, Volume 50, Issue 1, 2012, Pages $244-248$.

[29] ISO 1973:2021. Textile fibres - Determination of linear density - Gravimetric method and vibroscope method. Edition: 3 , Number of pages: 10, Publication date: 2021, ICS Code: 59.060.01 Textile fibres in general *Including mixtures of fibres.

[30] ASTM D1577-07(2018). Standard test methods for linear density of textile fibers. ASTM International, Book of Standards Volume: 07.01, Developed by Subcommittee: 58, Number of pages: 11, ICS Code: 59.060.01 Textile fibres in general *Including mixtures of fibres.

[31] P. Gonnet, Z. Liang, E.S. Choi, R.S. Kadambala, C. Zhang, J.S. Brooks, B. Wang, and L. Kramer. Thermal conductivity of magnetically aligned carbon nanotube buckypapers and nanocomposites. Current Applied Physics, Volume 6, Issue 1, 2006, Pages 119-122.

[32] J. Hone, M.C. Llaguno, N.M. Nemes, and A.T. Johnson. Electrical and thermal transport properties of magnetically aligned single wall carbon nanotube films. Applied Physics Letters, Volume 77, Issue 5, 2000, Pages 666-668.

[33] F.G. Emmerich. Young's modulus, thermal conductivity, electrical resistivity and coefficient of thermal expansion of mesophase pitch-based carbon fibers. Carbon, Volume 79, 2014, Pages 274-293.

[34] D. Jang and S. Lee. Correlating thermal conductivity of carbon fibers with mechanical and structural properties. Journal of Industrial and Engineering Chemistry, Volume 89, 2020, Pages 115-118.

[35] L.M. Ericson, H. Fan, H. Peng, V.A. Davis, W. Zhou, J. Sulpizio, Y. Wang, R. Booker, J. Vavro, C. Guthy, A.N.G. ParraVasquez, M.J. Kim, S. Ramesh, R.K. Saini, C. Kittrell, G. Lavin, H. Schmidt, W.W. Adams, W.E. Billups, M. Pasquali, W.-F. Hwang, R.H. Hauge, J.E. Fischer, and R.E. Smalley. Macroscopic, neat, single-walled carbon nanotube fibers. Science, Volume 305, Issue 5689, 2004, Pages 1447-1450.

[36] P. Liu, Z. Fan, A. Mikhalchan, T.Q. Tran, D. Jewell, H.M. Duong, and A.M. Marconnet. Continuous carbon nanotube-based fibers and films for applications requiring enhanced heat dissipation. ACS Applied Materials \& Interfaces, Volume 8, Issue 27, 2016, Pages 17461-17471.

[37] J. Qiu, J. Terrones, J.J. Vilatela, M.E. Vickers, J.A. Elliott, and A.H. Windle. Liquid infiltration into carbon nanotube fibers: Effect on structure and electrical properties. ACS Nano, Volume 7, Issue 10, 2013, Pages 8412-8422.

[38] A.E. Aliev, C. Guthy, M. Zhang, S. Fang, A.A. Zakhidov, J.E. Fischer, and R.H. Baughman. Thermal transport in MWCNT sheets and yarns. Carbon, Volume 45, Issue 15, 2007, Pages 2880-2888. 